$D O E / M C / 31190--1$

\title{
Coherent Laser Vision System (CLVS)
}

\author{
Topical Report \\ February 13, 1997
}

Work Performed Under Contract No.: DE-AC21-94MC31190

For

U.S. Department of Energy

Office of Fossil Energy

Federal Energy Technology Center

P.O. Box 880

Morgantown, West Virginia 26507-0880

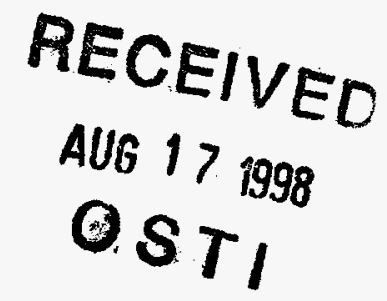

By

Coleman Research Corporation

Federal Systems Group 6551 Loisdale Court, Suite 800

Springfield, Virginia 22150

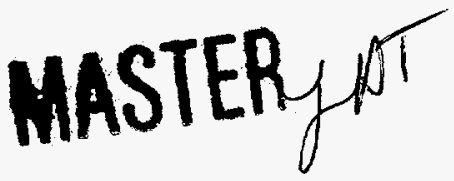




\section{Disclaimer}

This report was prepared as an account of work sponsored by an agency of the United States Government. Neither the United States Government nor any agency thereof, nor any of their employees, makes any warranty, express or implied, or assumes any legal liability or responsibility for the accuracy, completeness, or usefulness of any information, apparatus, product, or process disclosed, or represents that its use would not infringe privately owned rights. Reference herein to any specific commercial product, process, or service by trade name, trademark, manufacturer, or otherwise does not necessarily constitute or imply its endorsement, recommendation, or favoring by the United States Government or any agency thereof. The views and opinions of authors expressed herein do not necessarily state or reflect those of the United States Government or any agency thereof. 


\section{DISCLAIMER}

Portions of this document may be illegible electronic image products. Images are produced from the best available original document. 


\section{Table of Contents}

ABSTRACT $\ldots \ldots \ldots \ldots \ldots \ldots \ldots \ldots \ldots \ldots \ldots \ldots \ldots \ldots \ldots \ldots \ldots \ldots \ldots \ldots \ldots \ldots$

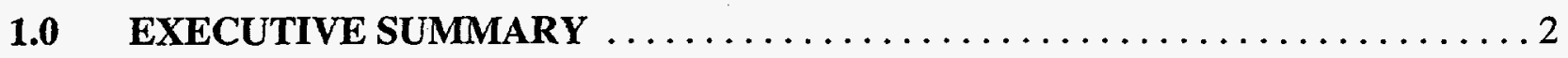

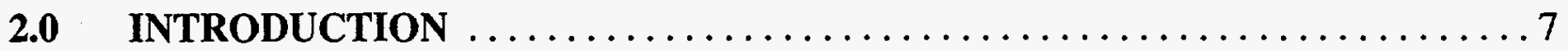

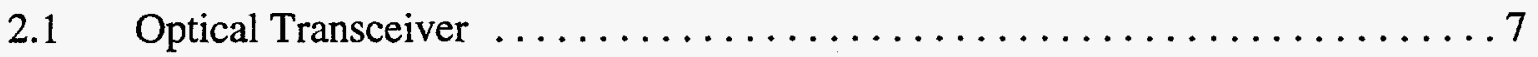

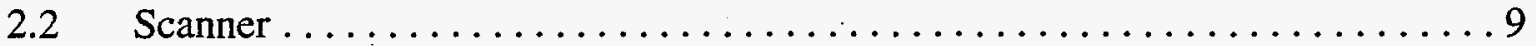

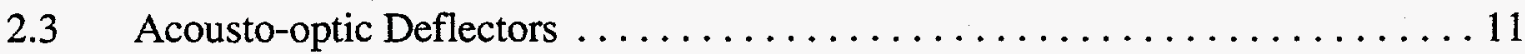

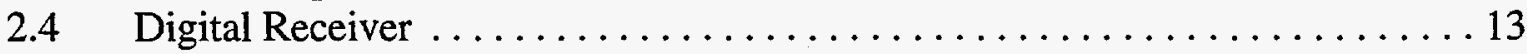

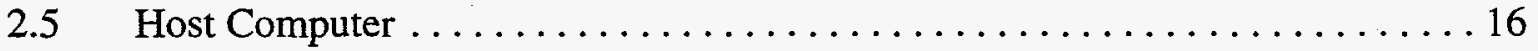

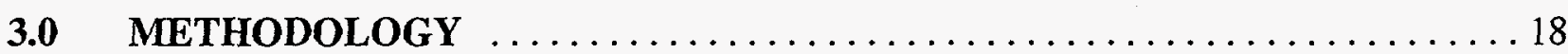

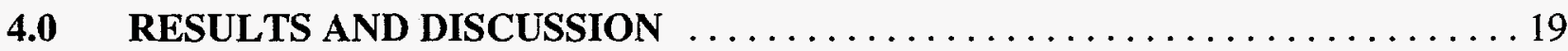

4.1 CLVS System Requirements and Specifications $\ldots \ldots \ldots \ldots \ldots \ldots \ldots \ldots$

$4.2 \quad$ Test Plan ............................................ 20

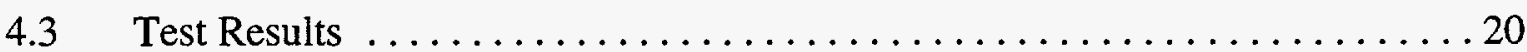

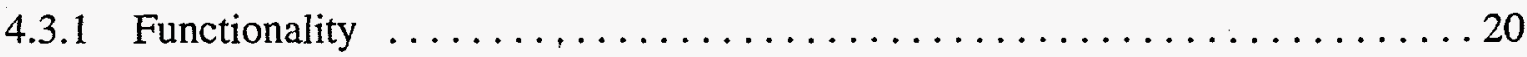

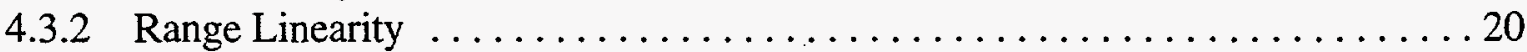

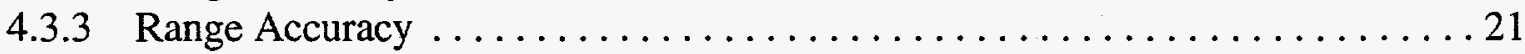

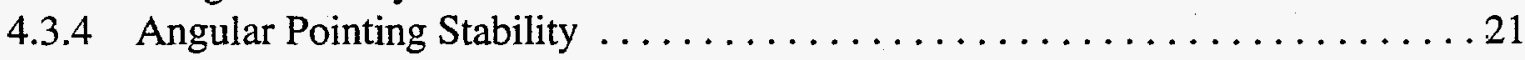

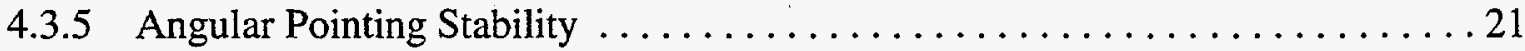

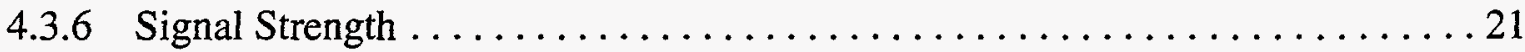

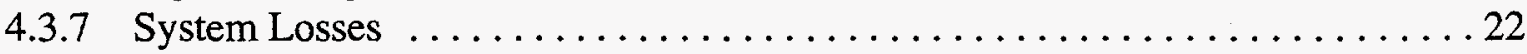

4.4 Demonstration $\ldots \ldots \ldots \ldots \ldots \ldots \ldots \ldots \ldots \ldots \ldots \ldots \ldots \ldots \ldots \ldots \ldots \ldots \ldots \ldots \ldots \ldots, 22$

5.0 CONCLUSIONS AND RECOMMENDATIONS $\ldots \ldots \ldots \ldots \ldots \ldots \ldots \ldots \ldots \ldots$

5.1 Conclusions Drawn From the Research Effort $\ldots \ldots \ldots \ldots \ldots \ldots \ldots \ldots$

5.2 Recommendations for Future Work ...................... 27

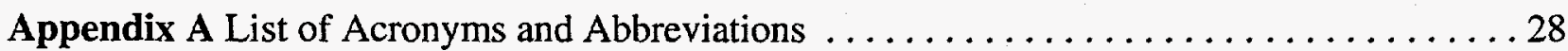

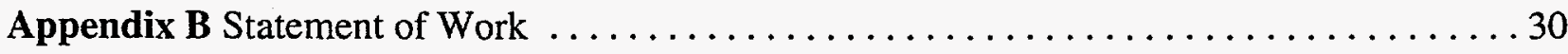




\section{Table of Contents (cont'd)}

\section{List of Figures}

Figure 2-1. CLVS Laser Radar Vision System .......................

Figure 2-2. Optical Transceiver and Scanner Block Diagram $\ldots \ldots \ldots \ldots \ldots \ldots \ldots$

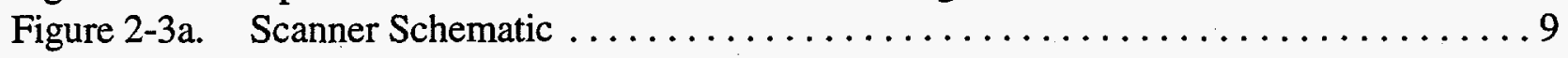

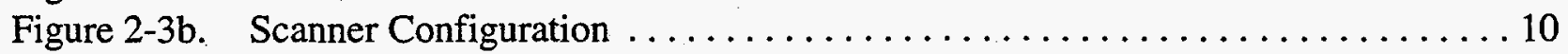

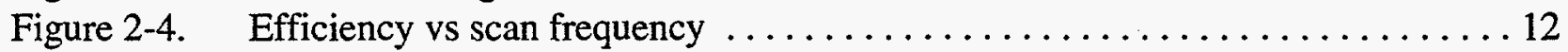

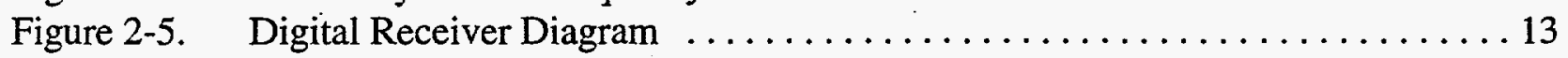

Figure 2-6. CLVS Signal Processing Flow ........................... 15

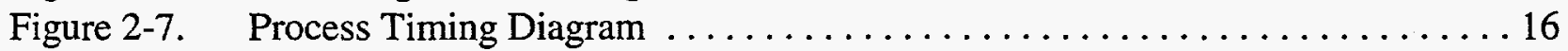

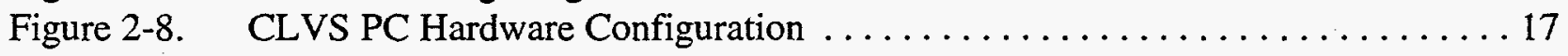

\section{List of Tables}

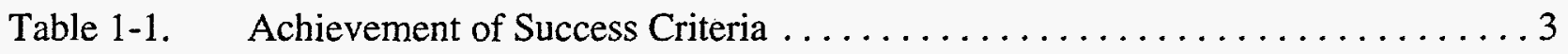

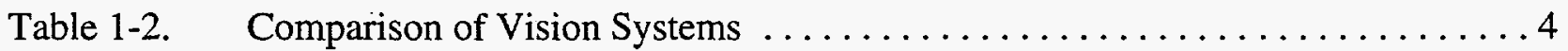

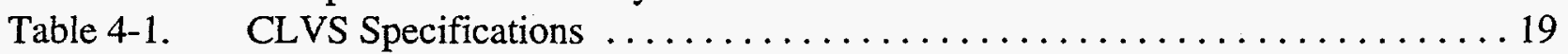

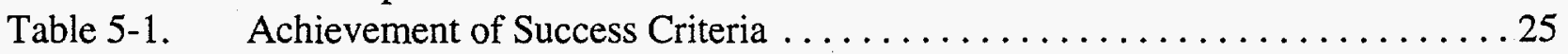

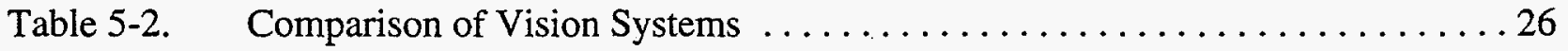




\begin{abstract}
The purpose of the CLVS research project is to develop a prototype fiber-optic based Coherent Laser Vision System suitable for DOE's EM Robotics program. The system provides three-dimensional (3D) vision for monitoring situations in which it is necessary to update geometric data on the order of once per second.

The CLVS project plan required implementation in two phases of the contract, a Base Contract and a continuance option. This is the Base Program Interim Phase Topical Report presenting the results of Phase I of the CLVS research project. Test results and demonstration results provide a proof-of-concept for a system providing three-dimensional (3D) vision with the performance capability required to update geometric data on the order of once per second.
\end{abstract}




\subsection{EXECUTIVE SUMMARY}

The purpose of the CLVS research project is to develop a prototype fiber-optic based Coherent Laser Vision System suitable for DOE's EM Robotics program. The system provides three-dimensional (3D) vision for monitoring situations in which it is necessary to update geometric data on the order of once per second. The CLVS project plan required implementation in two phases of the contract, a Base Contract and a continuance option.

In the Base Contract, development of a prototype Coherent Laser Radar 3D vision system with a no moving parts scanner, baseline frame size of $128 \times 128$ pixels, one frame per second with fixed focus 1.5 meter depth of range was accomplished.

In the continuance option, $\mathrm{CRC}$ will develop a compact scanner and implement advanced receiver algorithms to achieve a fieldable prototype coherent laser radar 3D vision system with an increased frame size of $256 \times 256$ pixels and an operating range beyond 5 meters.

The Base Contract phase has been successfully completed. The prototype system developed has been tested to system specifications and requirements and fully meets all specifications and requirements. Table 1 summarizes Coleman Research Corporation's achievement of success criteria listed in the Management Plan (Base Contract). In addition, all tasks in the Base Contract, i.e. tasks 1.1 through 1.7 inclusive, have been completed. As was shown in the laboratory, the CLVS meets all functional and technical performance requirements. There were no performance requirements on signal-to-noise (SNR) or image quality, but both were worse than expected, so although the system met all performance requirements, especially range accuracy and repeatability, accuracy measurements had to be made on very strongly reflective targets and real time image quality was not as good as desired. It should be emphasized that the laboratory test was run with the Bragg cells being driven at a very conservative 3 watts. At the end of the test, drive power was increased to 6 watts with noticeable improvement in image quality before one of the Bragg cells burned out. In summary, the laboratory system represents a significant step forward in vision systems and no moving parts scanners; all success criteria and performance requirements were satisfied; although SNR and image quality were much poorer than expected, the reasons are understood, and we have every expectation that the next generation of Bragg cell no moving parts scanner will perform as desired. 
Table 1-1. Achievement of Success Criteria

\begin{tabular}{||l|l|l||}
\hline Number & \multicolumn{1}{|c|}{ Criterion } & \multicolumn{1}{c|}{ Achievement } \\
\hline \hline 1 & $\begin{array}{l}\text { Developed CLVS produces 128 x 128 } \\
\text { pixel frame, one frame per second real- } \\
\text { time 3D images for 5m working range } \\
\text { (changed via contract modification to } \\
1.5 \text { meter depth of range) }\end{array}$ & $\begin{array}{l}\text { a. } 128 \times 128 \text { pixel frame. } \\
\text { b. One frame per 1.023 seconds } \\
\text { demonstrated; adjustable by count- } \\
\text { down factor to one frame per 1.0006 } \\
\text { seconds. } \\
\text { c. Real time 3D gray scale images } \\
\text { demonstrated. } \\
\text { d. Depth of range of 1.5 meter } \\
\text { demonstrated. }\end{array}$ \\
\hline Individual pixel range error & $\begin{array}{l}\text { Pixel range error shown by test } \\
\text { procedure to be less than 1 mm for } \\
\text { ap-sweeps and down-sweeps for } \\
\text { ranges in the close half of depth of } \\
\text { range, and for down-sweeps in the } \\
\text { far half of the depth of range. } \\
\text { Standard deviation ranges from less } \\
\text { than 1 mm to 3 mm for up-sweeps in } \\
\text { the far half of the depth of range. } \\
\text { This has been attributed to known } \\
\text { problems in the supplier's data } \\
\text { processing board and interface, } \\
\text { problems that are easily fixed. }\end{array}$ \\
\hline
\end{tabular}




\section{APPLICATIONS}

Table 2 shows a matrix comparing the CLR vision system with the CLR mapper and with other technology: Amplitude modulated (AM) vision; stereoscopic vision, and structured light. The table clearly points out the unique niche of the FM CLR Vision system for physically small, medium accuracy, high speed mapping that is insensitive to ambient lighting conditions.

Table 1-2. Comparison of Vision Systems

\begin{tabular}{|l|l|l|l|l|l||}
\hline & $\begin{array}{c}\text { FM CLR } \\
\text { Vision }\end{array}$ & $\begin{array}{c}\text { FM-CLR } \\
\text { Mapper }\end{array}$ & AM Vision & \multicolumn{1}{|c|}{$\begin{array}{c}\text { Structured } \\
\text { light }\end{array}$} & \multicolumn{1}{|c|}{$\begin{array}{c}\text { Stereo- } \\
\text { scopic }\end{array}$} \\
\hline \hline $\begin{array}{l}\text { Effect of } \\
\text { ambient light }\end{array}$ & No effect & No effect & Serious & Serious & Moderate \\
\hline Safety & Class I & Class I & Class III & Class III & N/A \\
\hline $\begin{array}{l}\text { Immunity of } \\
\text { range to shading } \\
\text { and color }\end{array}$ & Yes & Yes & No & No & No \\
\hline Speed & Fast & Slow & Fast & Moderate & Fast \\
\hline Sensor geometry & Single unit & Single unit & Single unit & $\begin{array}{l}\text { Multiple units } \\
\text { with large } \\
\text { baseline }\end{array}$ & $\begin{array}{l}\text { Multiple } \\
\text { sensors }\end{array}$ \\
\hline Range accuracy & Medium & V. High & Low & Medium & Low \\
\hline Angle accuracy & High & V. High & Low & Low & Low \\
\hline Depth of range & Medium & High & High & Medium & Medium \\
\hline Calibration & Auto & Auto & External & Difficult & Difficult \\
\hline Field of view & Small & Large & Large & Large & Medium \\
\hline
\end{tabular}

The main advantage of the CLVS is its ability to produce moderately accurate range maps over a limited volume very fast with automatic calibration and under any and all lighting and surface shading conditions. Major applications are:

- Fast coarse facility mapping, possibly as a prelude to slow, highly accurate, high density mapping by the CLR mapper. 
- $\quad$ Fast high density 3D mapping of a restricted volume as encountered in glove box operations, part identification and determination of location and orientation.

- Monitoring a waste stream, utilizing a single dimension scan of the waste stream passing the sensor unit.

\section{PRINCIPLES OF OPERATION}

The CLVS combines CRC's coherent laser radar range measurement technology with a unique solid state scanner developed under this program. Range is measured by a frequency modulated infrared laser. The transmitted waveform is a linear frequency modulated chirp. When mixed with the returned energy reflected by a target a constant frequency signal is generated. The frequency of this signal is proportional to range to the target. The wide bandwidth of the transmitted waveform, $25-100 \mathrm{GHz}$., and coherent processing by the receiver provide the ability to measure off of any surface, excellent accuracy, and total immunity to ambient lighting conditions.

Beam pointing is commonly achieved by using a two-axis mirror to direct the laser beam. Though accurate, these scanners are typically large, have moving parts, and too slow for vision systems in which the beam must be repositioned in microseconds. The goal of this project was to develop a small, no moving parts, fast solid state scanner. The approach was to design a twodimensional scanner using a set of two crossed one-dimensional Bragg cell beam directors. One cell steers the beam in azimuth while the second cell steers the beam in elevation. Each individual cell operates in the same fashion. The laser beam is directed onto one side of the Bragg cell, passes through the cell, and exits the other side along a different direction. The angle of deflection of the beam as it passes through the cell can be controlled within limits by producing a standing vibrational wave in the crystal; the angle of deflection is determined by the frequency of the vibration. This is achieved by attaching a transducer to the end of the crystal that converts an electrical input signal into a mechanical vibration in the crystal.

\section{DEMONSTRATION}

A program briefing with laboratory demonstration was held at Coleman Research Corp's Springfield Virginia facility on 13 November 1996. The purpose of the laboratory demonstration was to show the real time image display capability under various conditions of range, lighting, and target characteristics. The Bragg cells were driven at a conservative $3 \mathrm{~W}$ during the demonstration to avoid damage. The system displayed reasonably good images at 128 by 128 pixel frames, one frame per second, over a depth of range of 1.5 meters, under lighting conditions ranging from no lights through normal lab lighting to having a spotlight shining directly into the scanner. All functional specifications were met. However, image quality was not as good as desired because signal losses in the Bragg cells were much higher than expected. Since losses could be reduced by driving the Bragg cells at higher RF power, at the end of the demonstration, when damage to the 
Bragg cells would not be catastrophic, the Bragg cell drive power was changed. With an image being displayed, the drive power to each cell was reduced from $3 \mathrm{~W}$ to $1.5 \mathrm{~W}$. The result was a significantly degraded image. Then the drive power was increased to $6 \mathrm{~W}$. The result was a significantly improved image. The conclusion is that improved Bragg cell fabrication providing losses in agreement with design expectations will significantly improve image quality. 


\section{$2.0 \quad$ INTRODUCTION}

The Coherent Laser Radar Vision System (CLVS) system consists of: optical transceiver, scanner, digital receiver, and video monitor shown in Figure 2-1. The system is controlled by a host computer which is not shown.

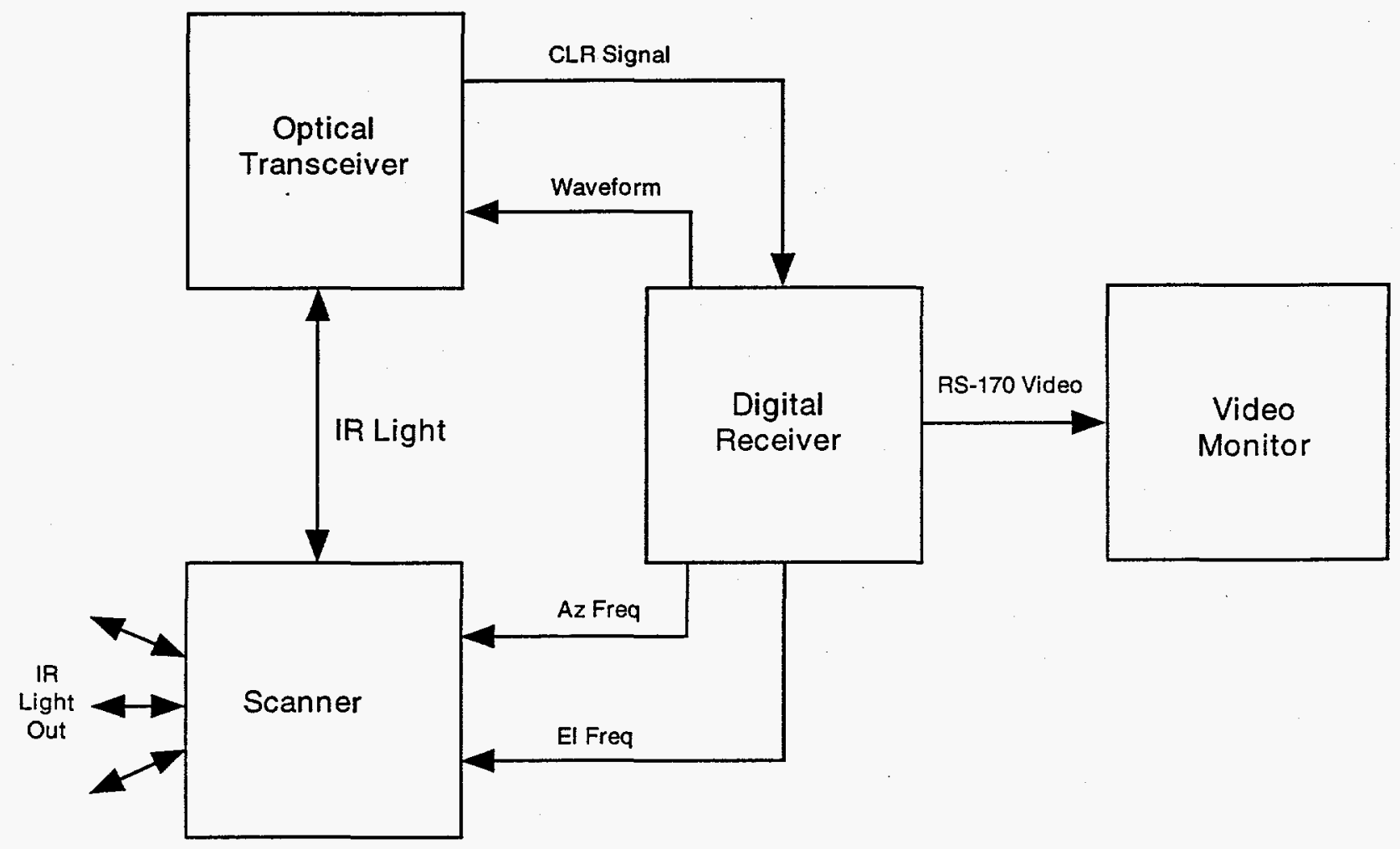

Figure 2-1. CLVS Laser Radar Vision System

\subsection{Optical Transceiver}

The laser transmitter is based on a fiber pigtailed laser diode which is frequency modulated by varying the laser drive current. The linear frequency modulated (FM) chirp drive waveform is supplied by the digital receiver. The waveform is designed to produce a linear FM chirp of $25 \mathrm{GHz}$. in $61 \mu \mathrm{sec}$. At one chirp per pixel, the system provides one frame per second at $128 \times 128$ pixels per frame. Fast, wideband FM waveforms are desired because range is inversely proportional to the FM chirp rate. The entire optical transceiver, Figure 2-2, is implemented using fiber-optics except for the input drive waveform and the pinFET detector output signal which is at radio frequency (RF). 
A small portion of the laser's output is directed into the local oscillator (LO) delay path via the LO power tap. The remainder of the light is amplified and passed through a fiber optic circulator and polarization rotator and then to the acousto-optic (AO) scanner. Light reflected from a surface is collected by the scanner and returned to the optical transceiver via the polarization rotator and optical circulator. The returned signal and the LO delay signal are mixed in the mixing coupler. The mixed IR signal is passed through the isolator and directed onto the pinFET photodiode detector. The output from the photodiode detector is an RF signal which is passed to the digital receiver for processing to estimate range to the reflecting surface.

The LO delay is simply a length of optical fiber of specified length. When the IR signal passes through the LO delay fiber, it is delayed proportional to the length of the fiber. The main signal is then combined with the delayed signal in the mixer producing sum and difference frequency terms. The difference frequency terms are passed by a low pass filter. Consequently, the process is similar to a heterodyne receiver in which the heterodyning signal is provided by a local oscillator, hence the use of the term LO for the optical delay line.

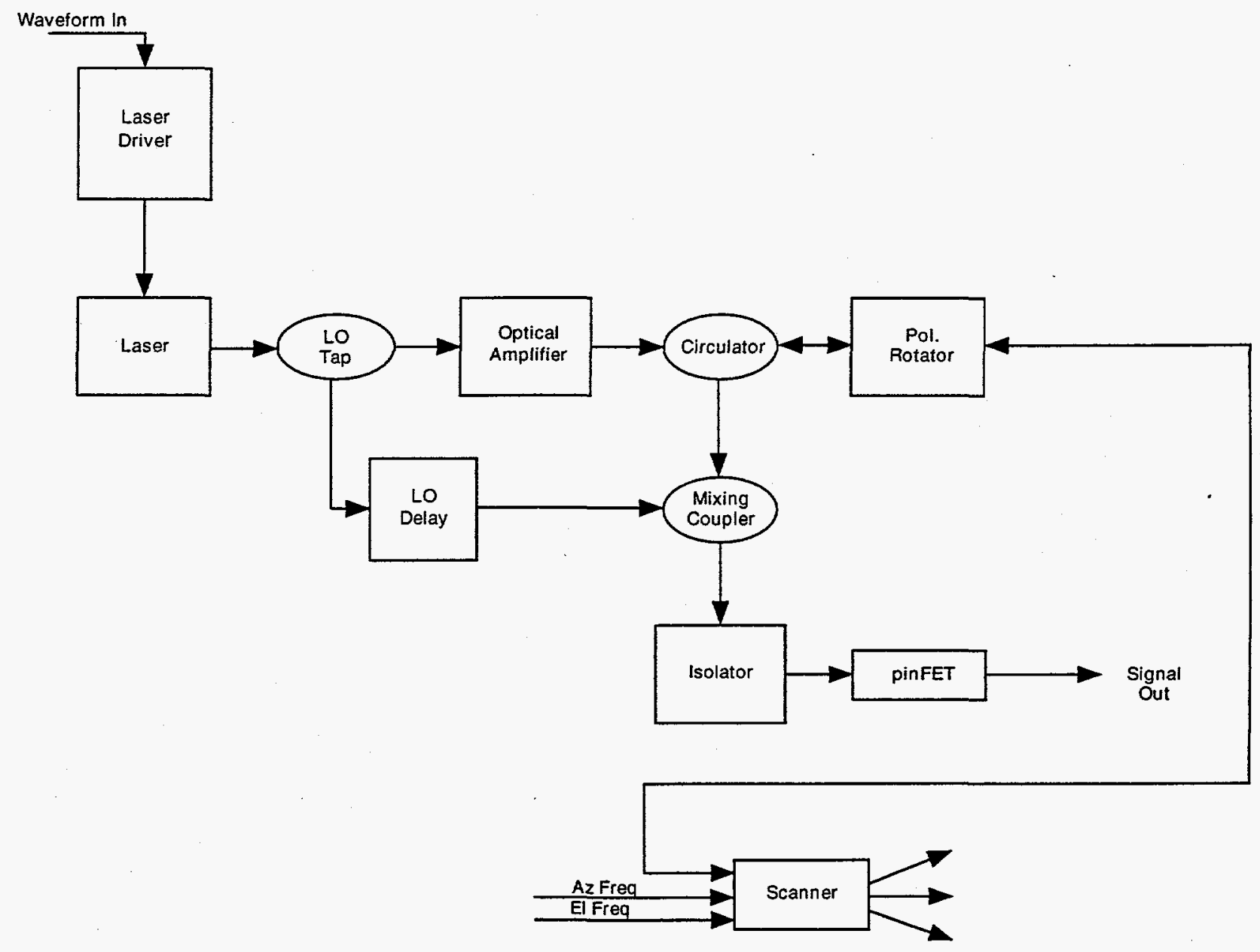

Figure 2-2. Optical Transceiver and Scanner Block Diagram 


\subsection{Scanner}

Figure 2-3a is a schematic of the AO scanner designed for the CLVS. The approach to the implementation of a rapid $\mathbb{R}$ scanner was to use two acousto-optic Bragg cells (discussed in section 2.3) to deflect the laser beam in the focal plane of a wide-angle scan lens where a $128 \times 128$ lenslet array is positioned. Thus the scanner addresses individual lenslets in a lenslet array. This approach exploits the rapid access and high light throughput efficiency of AO scanners while maintaining the wide angular field of view achievable with the large aperture scan lens.

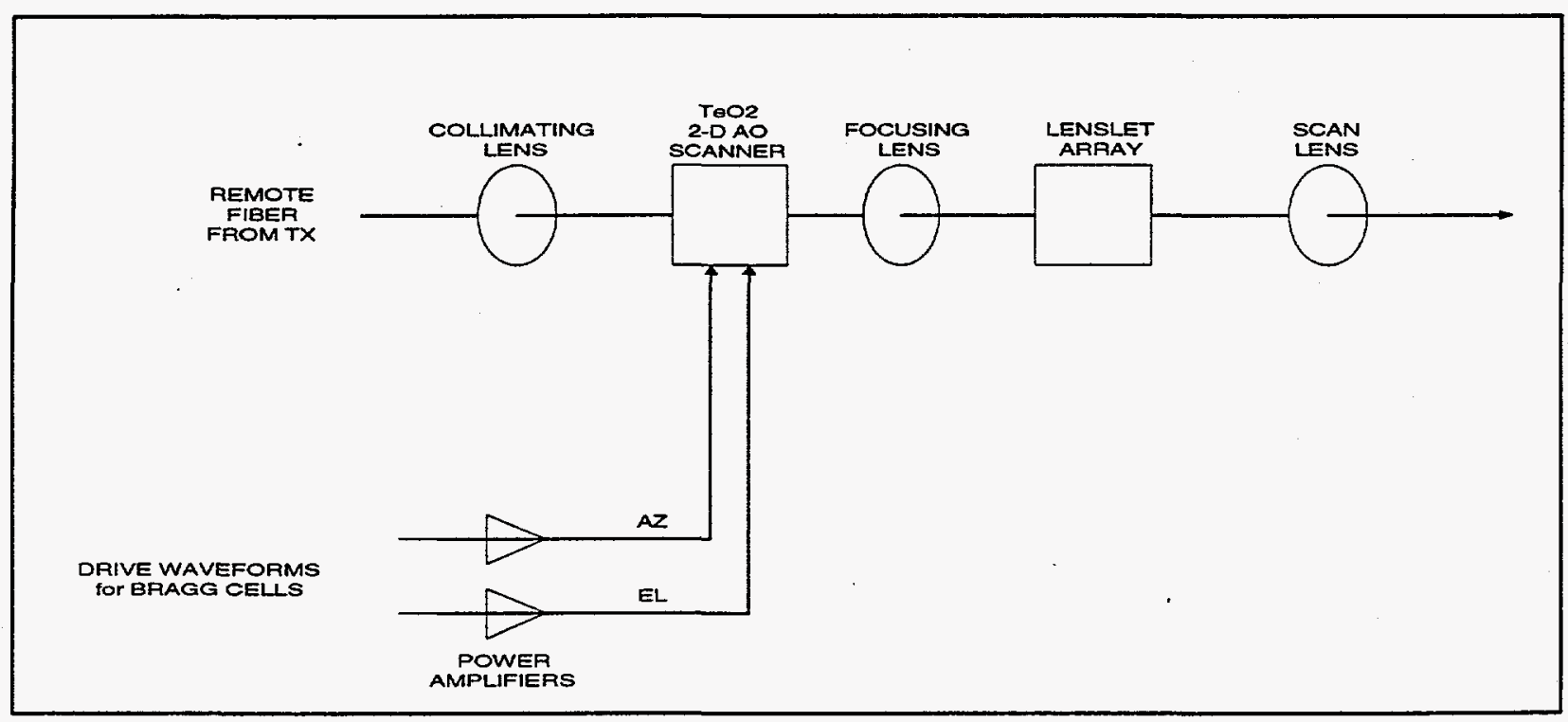

Figure 2-3a. Scanner Schematic

CRC has developed a fully optimized 2D acousto-optic scanner for the infrared (IR) wavelength regime that utilizes a $128 \times 128$ lenslet array to achieve rapid $(10 \mu \mathrm{sec})$ scanning over a full 20 degree field of view, while maintaining a large $(1 \mathrm{~cm})$ collection aperture.

The light from the input fiber is collimated, and illuminates a 2-dimensional wide bandwidth Tellurium dioxide $\left(\mathrm{TeO}_{2}\right) \mathrm{AO}$ deflector at the Bragg angle ${ }^{1}$. The diffracted light is focused with a long enough focal length lens to illuminate the full scan lens input aperture, which is about $1 \mathrm{~cm}$. At this input plane is placed a two-dimensional lenslet array containing $128 \times 128$ lenslets that further reduce the spots produced by the $\mathrm{AO}$ deflector to the spot size required to fully illuminate the scan lens aperture, thereby achieving the full resolution and deflection angle capabilities of the scan lens.

Beam pointing is achieved by driving each of the two Bragg cells with an RF sinusoidal signal of appropriate frequency. These azimuth and elevation beam deflection control signals are

\footnotetext{
${ }^{1} \mathrm{Bragg}$ angle $=$ angle light enters $\mathrm{AO}$ modulator in order to maximize throughput.
} 
provided by the digital receiver. A high addressing speed of $10 \mu \mathrm{sec}$ is achievable with $50 \mathrm{MHz}$ bandwidth $\mathrm{TeO}_{2} \quad \mathrm{AO}$ deflectors. Beam deflection is proportional to the frequency of the drive waveform. Efficiency, i.e. IR signal attenuation, is proportional to the RF signal power. The design specified operating the Bragg cells at 6 watts of RF drive power where throughput losses were expected to be $9-12 \mathrm{~dB}$.

The short $(6-8 \mathrm{~mm})$ cell allows a circular aperture to be utilized to implement a compact 2D scanner with a penalty factor of about 6 in efficiency, requiring about 6 watts of RF drive. High throughput approaching as little as $9-12 \mathrm{~dB}$ is achievable since the entire circular beam from the collimated fiber can be diffracted without truncation, and acoustic loss is very small for this short aperture. Since the height to acoustic aperture ratio in this case is about 6 times larger than necessary, as dictated by the near field condition in the height dimension, the entire device is 36 times closer to the near field. This dramatically improves the homogeneity of the diffracting acoustic field, which improves the diffracted beam quality and the corresponding fiber coupling efficiency.

It was discovered that the shape of the beam at the output of the Bragg cell deflector was not ideal and was not matched properly to the lenslets, producing losses that were greater than expected. Consequently, a second scanner configuration shown in Figure 2-3b was examined. This scanner did not use the lenslet array and, as a consequence of not using the lenslet array, did not use the output scan lens, thereby reducing the angular field of view about 2:1.

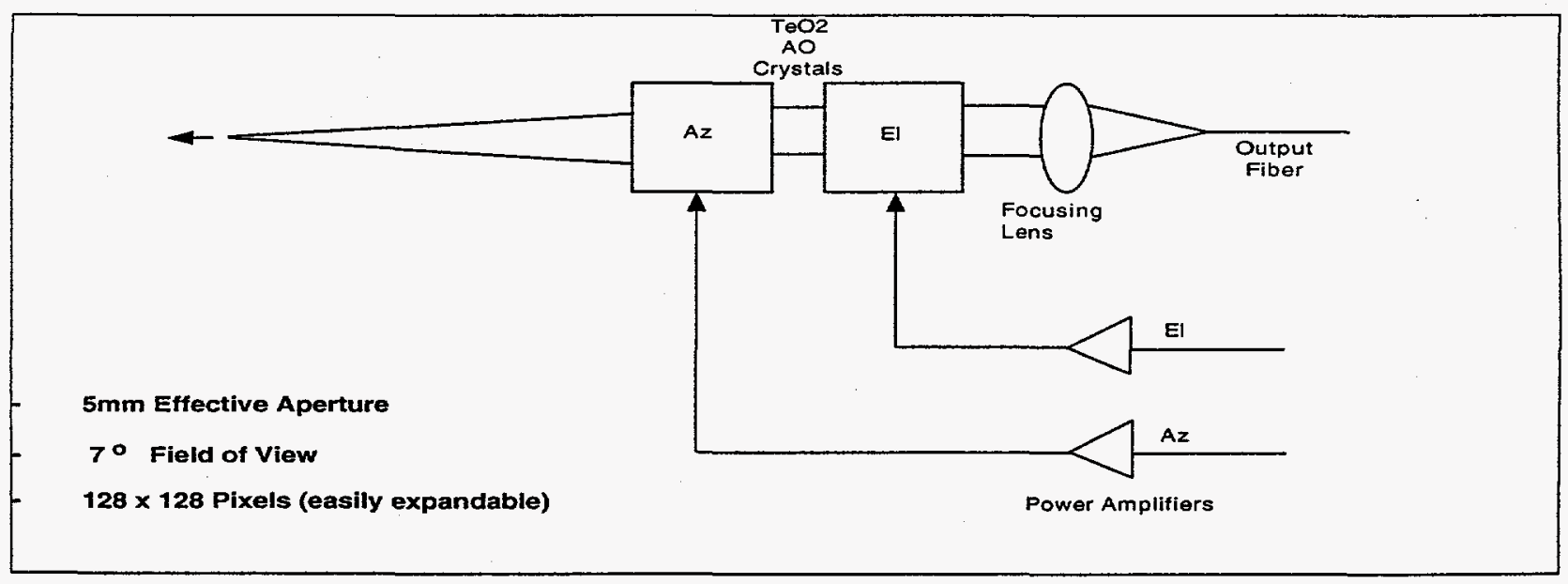

Figure 2-3b. Scanner Configuration 


\subsection{Acousto-optic Deflectors}

The optimization of the acousto-optic device was intended to achieve the highest possible diffraction efficiency over the entire $1540-1565 \mathrm{~nm}$ wavelength range while still allowing the addressing of the necessary 128 spots within $10 \mu \mathrm{s}$. For the 2-D crossed AO device scanner, it was found that a non-rotated phased-array device can be used in both stages. The design requires 2.35 watts of acoustic power to be launched into the desired beam steering order to achieve $80 \%$ diffraction efficiency. On $\mathrm{TeO}_{2}$ devices one can almost always use a four element tuner to achieve a voltage standing wave ratio (VSWR) of 1.5 over the entire band, but manufacturers are rarely willing to go beyond a VSWR of 2 , which corresponds to $5 \mathrm{~dB}$ of insertion loss. This means that 5.5 watts of RF power must be applied to launch the necessary 2.35 watts of acoustic power into the desired beam steering order to achieve $80 \%$ diffraction efficiency. To go from $80 \%$ to $100 \%$ diffraction efficiency requires a doubling of the acoustic power, since this is in the regime of diminishing return of the $\sin ^{2}$ diffraction efficiency. Now, the safe power that can be applied to a $\mathrm{LiNbO}_{3}$ transducer properly bonded with the correct thickness and impedance metal layer is 1 watt $/ \mathrm{mm}^{2}$. For this device the transducer is about $1.3 \mathrm{~mm}$ long and $6.5 \mathrm{~mm}$ high and the phased array duty factor is $75 \%$, giving a total transducer area of $6.3 \mathrm{~mm}^{2}$, presenting no problem to achieving in excess of $80 \%$ diffraction efficiency for each stage using a 6 watt amplifier at under $1 \mathrm{~W} / \mathrm{mm}^{2}$ power density on the transducer.

At these $\mathbb{R}$ wavelengths, this appears to be the optimal design for achieving high throughput efficiency in a two stage crossed AO deflector. The calculated efficiencies when 5 watts of RF power are applied as a function of the two frequencies are shown for three representative wavelengths in Figure 2-4. These excellent results show that with the design only 5 watts need to be applied to each $\mathrm{AO}$ device to achieve better than $3 \mathrm{~dB} A O$ inefficiency throughout the range of $52-98 \mathrm{MHz}$ on the first stage and $55-95 \mathrm{MHz}$ on the second stage. In addition this design achieves less than $6 \mathrm{~dB}$ loss throughout the full $50-100 \mathrm{MHz}$ frequency range at this safe 5 watts power level.

Acoustic absorption in the crystal results in heating of the $\mathrm{TeO}_{2}$ which can lead to thermal distortions that can affect the diffracted beam quality, and lead to additional power dependent tilt due to the inhomogeneous $\left(e^{-\alpha L}\right)$ heat source profile. At the low end of the frequency spectrum $(50 \mathrm{MHz})$ the acoustic loss in only $0.45 \mathrm{~dB}$, while at the high frequency end the acoustic loss is $1.8 \mathrm{~dB}$. This corresponds to only $10 \%$ of the applied power or 0.5 watt being absorbed at $50 \mathrm{MHz}$, while $33 \%$ or 1.7 watts are absorbed at $100 \mathrm{MHz}$. To dissipate this absorbed power good heatsinking is required, and to dissipate the transmitted power good coupling to an absorbing acoustic terminator is required. 


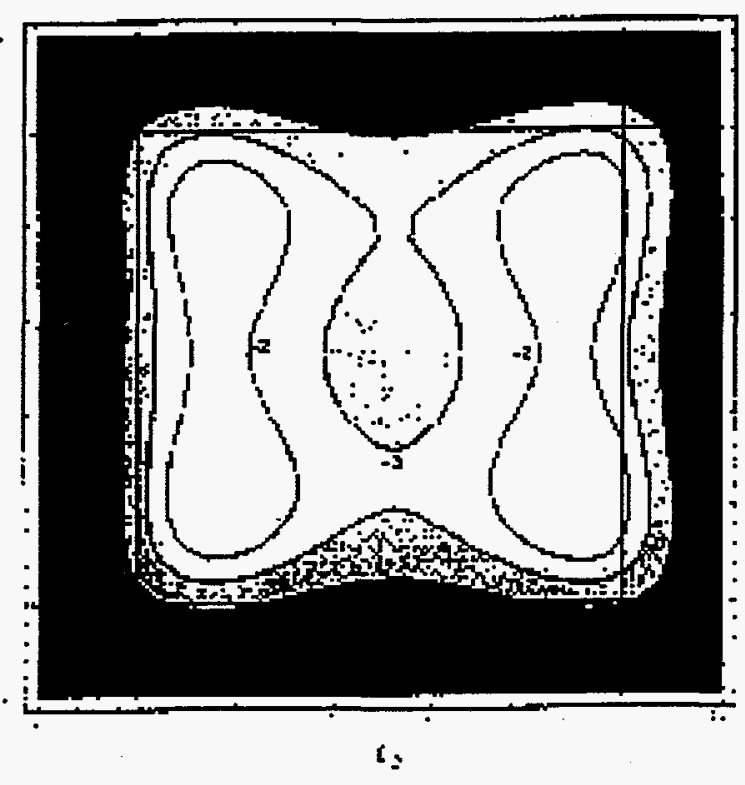

a j1.54 $\mathrm{cms}$

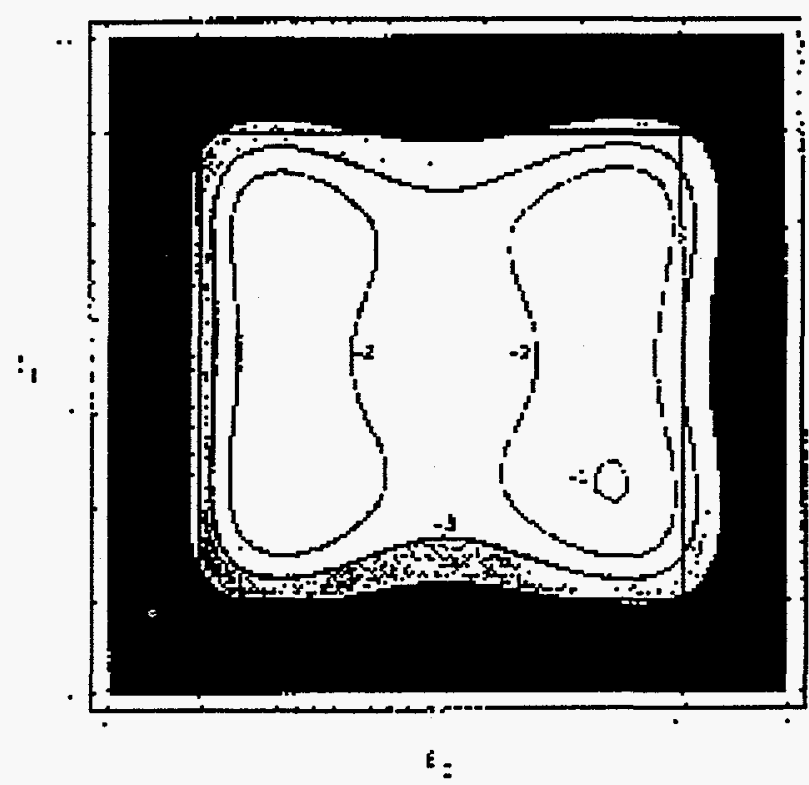

b) $1.5 \bar{s}$ ณn

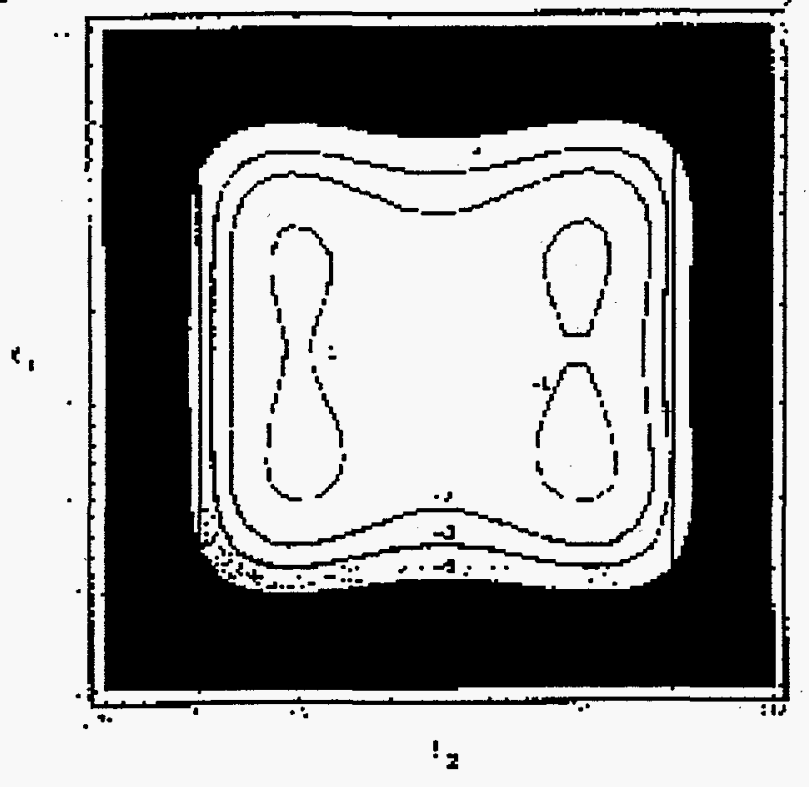

$\Rightarrow 1.565 . \mathrm{cm}$

Figure 2-4. Dependence of the throughput efficiency on the two scan frequencies $f 1$ and $f 2$ between 40 and $110 \mathrm{MHz}$ for 5 watts applied to each $\mathrm{AO}$ deflector at the 3 optical wavelengths a) $1.54 \mu \mathrm{m}$, b) $1.55 \mu \mathrm{m}$, c) $1.565 \mu \mathrm{m}$. The contours of acousto-optic throughput loss when driven by 5 watts $R F$ are $-1 \mathrm{~dB},-2 \mathrm{~dB}$ and $-5 \mathrm{~dB}$ (black). 


\subsection{Digital Receiver}

The Digital Receiver computes a range estimate for every pixel of every frame. Since the IR signal is a linear FM chirp, mixing the transmitted and returned IR signal in the pinFET detector produces a sinusoidal RF signal. The frequency of this sinusoidal signal is proportional to range. Hence the digital receiver estimates the frequency of the RF signal at the output of the pinFET detector. Processing must keep up with the $61 \mu \mathrm{sec}$ per pixel data rate.

The digital receiver estimates signal frequency, and the associated range, for each pixel by the following steps:

1) mix input signal to baseband in quadrature

2) digitize signal

3) compute 512 point FFT

4) estimate frequency of peak FFT magnitude

5) correct for LO delay offset and convert to range

6) insert range into the output frame

The Digital Receiver configuration is shown in Figure 2-5. The transmitted and reflected laser signals are mixed in the PinFET detector producing sum and difference frequency RF signals. The bandpass filter isolates the difference frequency RF signal components in the 150 to $450 \mathrm{MHz}$ range.

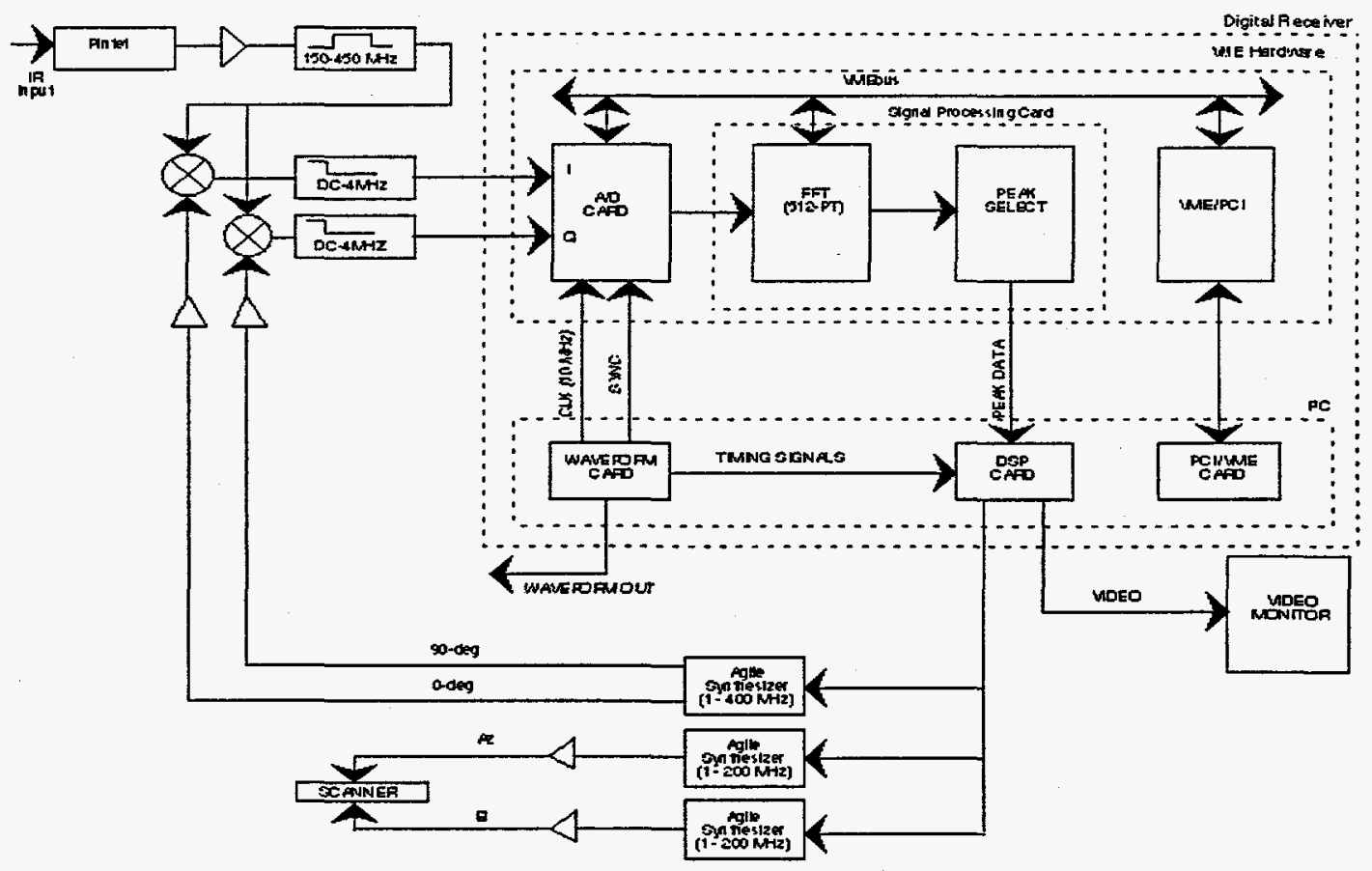

Figure 2-5. Digital Receiver Diagram 
The real analog bandpass signal converted to a complex sampled baseband signal using a quadrature sampler. The bandpass signal is mixed with a sinusoidal signal from the agile synthesizer, passed through a $4 \mathrm{MHz}$ low pass filter, and sampled at $10 \mathrm{MHz}$ to produce the inphase component. The bandpass signal is similarly processed with a sinusoidal signal of the same frequency but with 90 degree relative phase to produce the quadrature component. These two DC-4 $\mathrm{MHz}$ signals constitute a $-4 \mathrm{MHz}$ to $4 \mathrm{MHz}$ bandwidth complex data stream. The frequency of the agile synthesizer is determined by the frequencies used to drive the scanner because the Bragg cells impart a frequency shift on the IR that depends on the frequency of the RF drive.

Special purpose hardware computes a 512 point complex FFT, converts to magnitude, picks the peak magnitude and sends the magnitude of the FFT peak and two bins on either side to the PC for interpolation. The PC estimates the frequency by interpolating in the five values. The estimated frequency is converted to range and formatted for output. The signal processing flow is illustrated in Figure 2-6.

The A/D acquisition starts upon receipt of a Sync signal from the Waveform card in the PC. The Sync signal is generated once per pixel. Acquisition of 512-samples requires $51.2 \mu \mathrm{sec}$. The 512-point complex FFT requires approximately $50 \mu \mathrm{sec}$ and the peak-pick requires approximately $25 \mu \mathrm{sec}$. CLR data acquisition and signal processing are pipelined operations. This pipelined operation allows data acquisition and processing to occur simultaneously. Overall CLVS system timing is shown in Figure 2-7. 


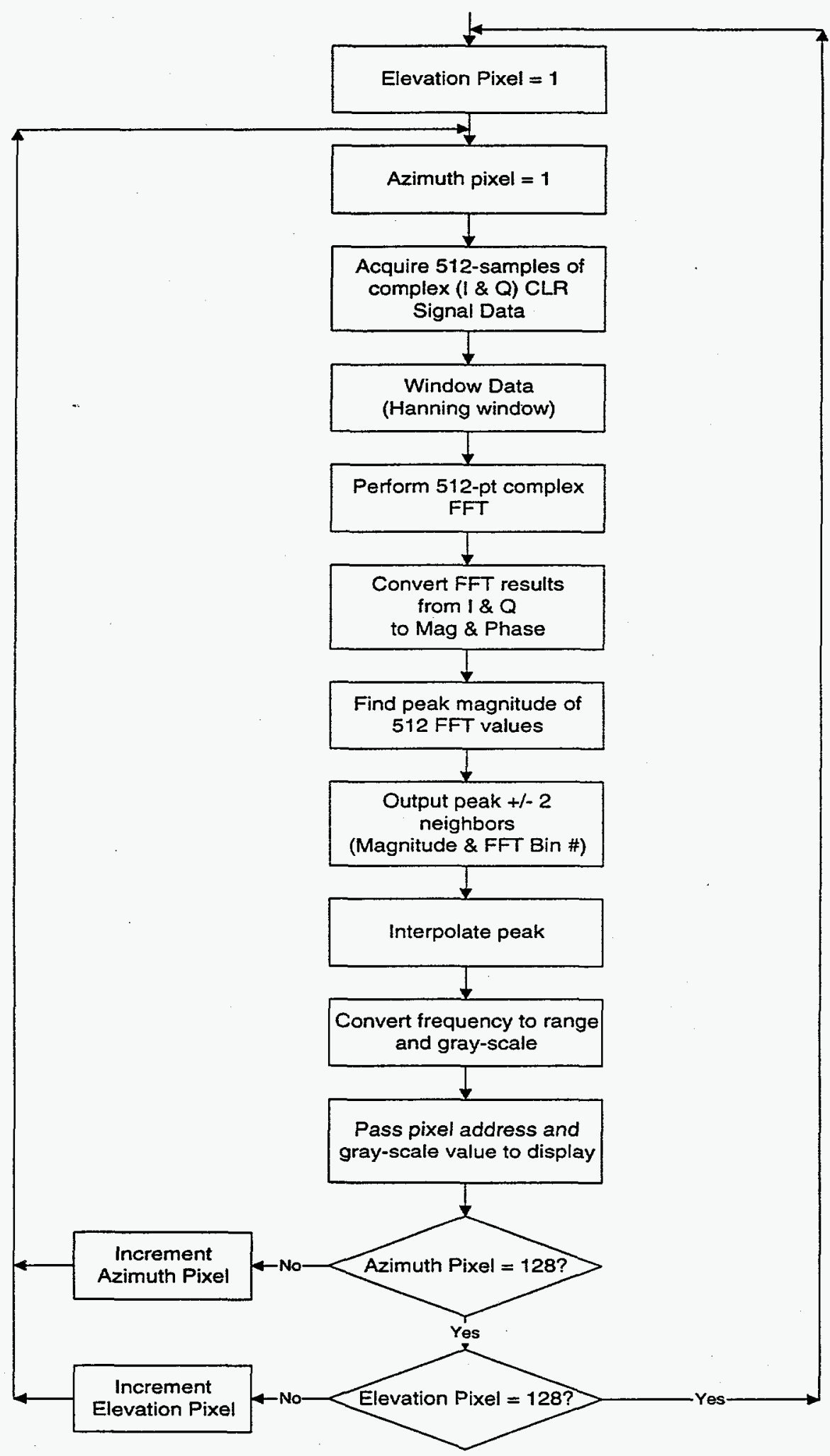

Figure 2-6. CLVS Signal Processing Flow 


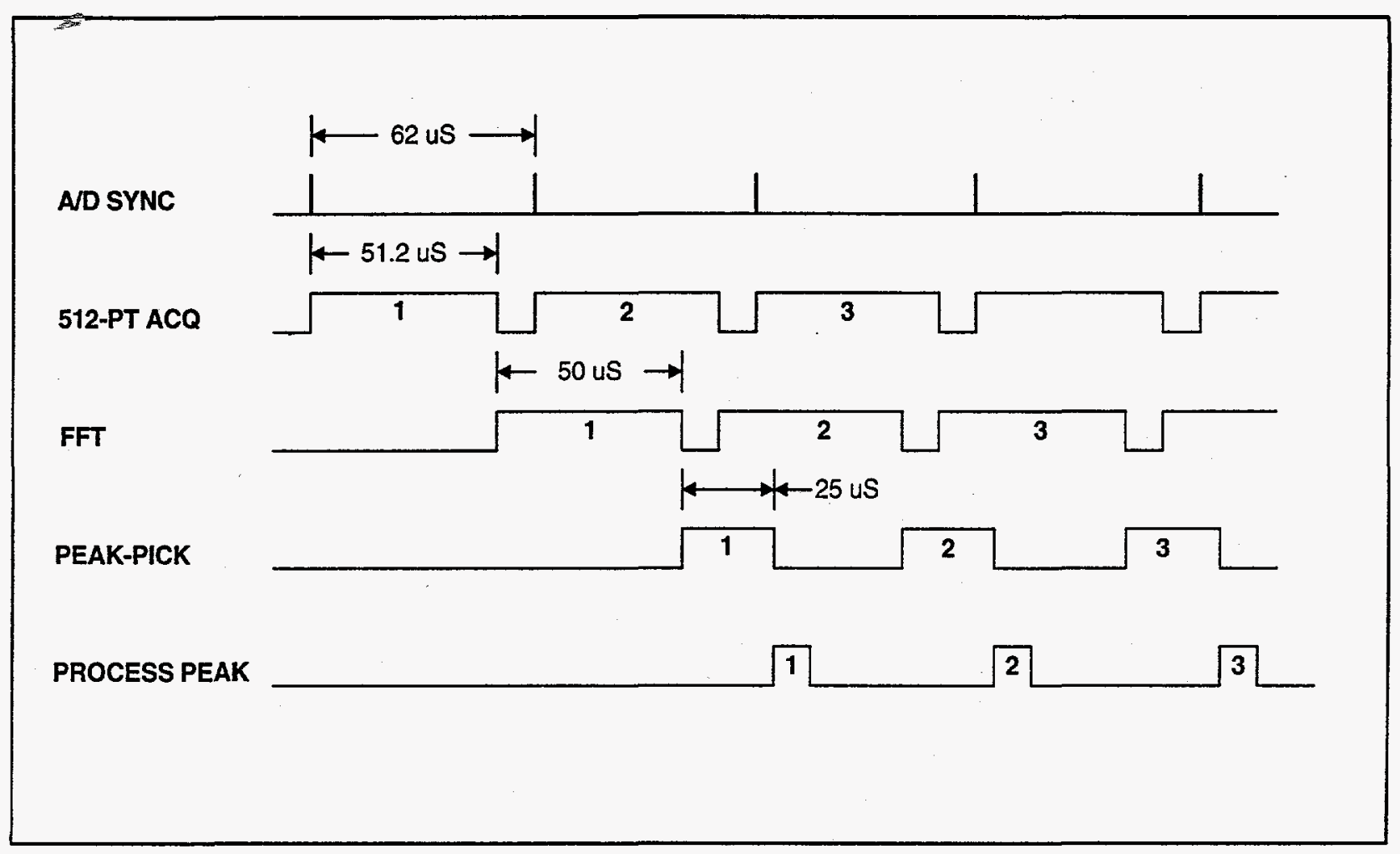

Figure 2-7. Process Timing Diagram

\subsection{Host Computer}

CLVS system control and graphical user interface software is installed and implemented on PC based hardware. The PC hardware configuration is shown in Figure 2-8. The PC configuration consist of: CPU, Waveform card, DSP card, and PCI/VME card. The PC operating system is OS/2.

The OS/2 operating system was used to allow use of existing CRC application software written for other existing CLR type systems. The CLVS graphical user interface (GUI) command and control software was derived from other existing CLR application software.

The Waveform card outputs a sample clock (10 MHz) to the Digital Receiver A/D converters and generates timing signals to the DSP card. These timing signals establish the CLVS frame rate. The DSP card consists of two DSP processors (TMS320C40). The C40_AV processor; acquires peak signal data from the Digital Receiver, controls Scanner frequencies for pixel selection, controls Digital Receiver front end frequency translation, and outputs 3D image data to the C40_BV processor for display on the video monitor. The C40_BV processor; acquires 3D image data from 
the C40_AV processor, loads pixel data into the pixel frame, converts the video frame to RS-170 video format, and outputs video data to the video monitor.

The PCI/VME card provides a link between the PCS PCI bus and the Digital Receiver VME bus. This PCI/VME interface is used to load and configure the Digital Receiver hardware and software as required by the CLVS system.

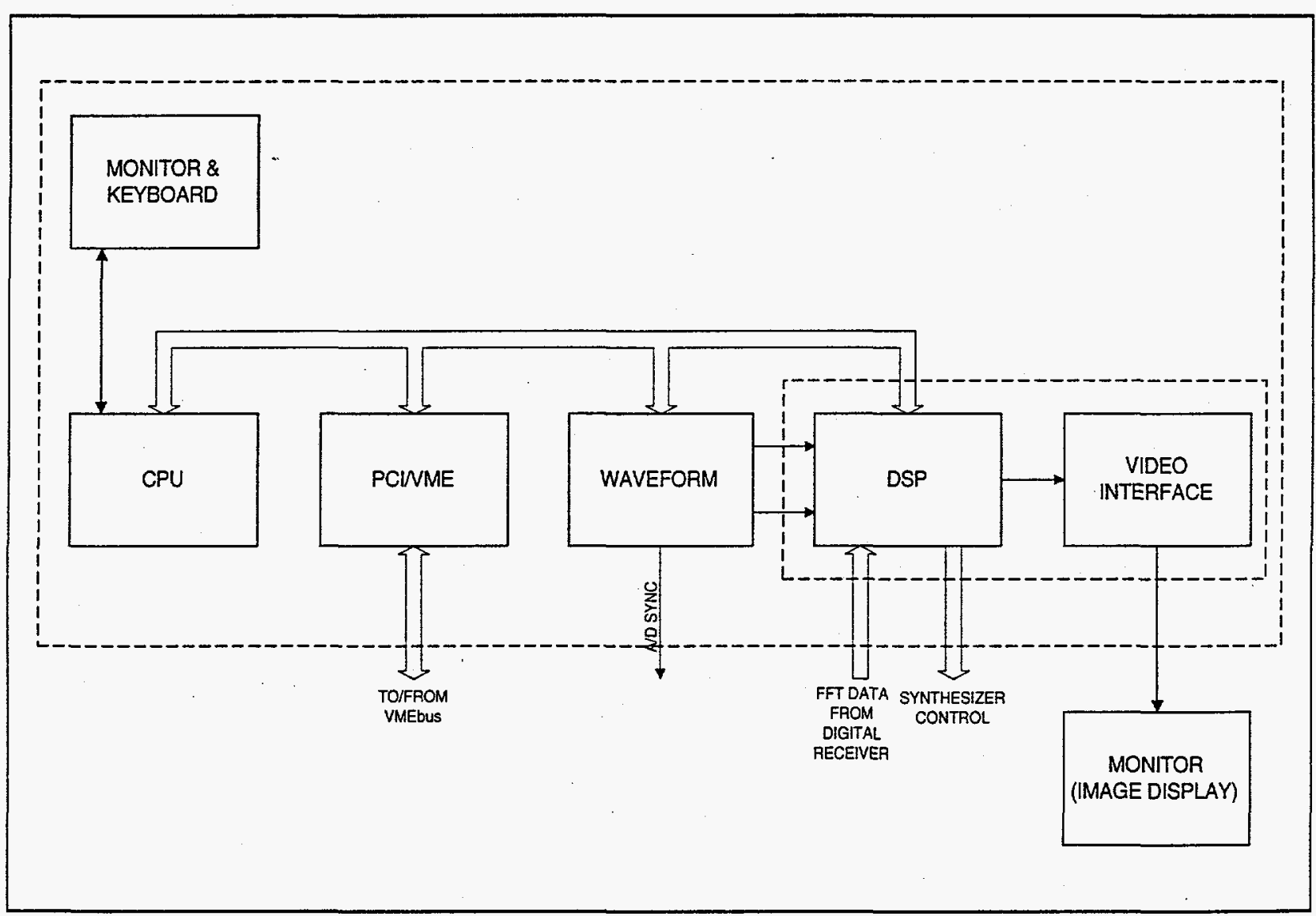

Figure 2-8. CLVS PC Hardware Configuration 


\subsection{METHODOLOGY}

The methodology for development has been to utilize CRC's existing fiber-optic coupled frequency modulated continuous-wave coherent laser radar technology, new solid-state scanner technology, and modified CRC laser radar system software to the maximum extent possible. This requires operation in the $\mathbb{R}$ in the region of $1550 \mathrm{~nm}$ wavelength $A O$ devices have higher efficiency in the visible spectrum, so $\mathbb{R}$ operation requires more RF drive power, raising concerns of device heating, blowing out the transducer, and amplifier cost. On the other hand, IR operation results in larger scan angles, in this case as large as 6 degrees, which is excellent for a large angle scanner, but is taxing on the optical design for diffraction limited performance. The wavelengths generated by the laser diodes used in the CLVS can vary from $1500 \mathrm{~nm}$ to $1585 \mathrm{~nm}$ with most of the devices in the $1540 \mathrm{~nm}$ to $1565 \mathrm{~nm}$ range. Thus the AO device must operate throughout this range. For the 2-D scanner a non-rotated design was settled on using a beamsteering transducer. The performance is essentially unchanged for a few nm of sweep of the chirped laser.

The main development problem involving the AO deflectors was the unexpectedly low diffraction efficiency. The most significant reason for this performance is that the manufacturer could not accommodate the necessary thicknesses of the bonding layers desired. It was calculated that for the $163^{\circ} \mathrm{Y}$-cut $\mathrm{LN} / \mathrm{TeO}_{2}$ combination (chosen for its thermal expansion-matching properties which enable potentially high-power operation), a single anti-reflection layer of Indium will produce a good transducer response. The manufacturer said that they make a Silver-Indium two-layer and could not change their technical routine for only two devices. Through subsequent analysis, it was calculated that a Silver-Indium bonding layer with a thickness of $5.6 \mu \mathrm{m}$ for the silver layer would produce excellent transducer response. Unfortunately, the manufacturer said that they could achieve a maximum silver thickness of $4 \mu \mathrm{m}$. Through more calculations it was determined that with an increase in the anti-resonant frequency of the transducer, acceptable result for a $4.1 \mu \mathrm{m}$ silver layer could be produced, although the high-frequency response is impacted. Unfortunately, the actual thickness finally realized by the manufacturer for the cells was only $3 \mu \mathrm{m}$.

These developmental problems led to a reassessment of the testing methodology in that two scanner geometric data were eventually investigated. Beside the geometry that utilizes the lenslet array scan lens combination described previously, testing was also performed on a simplified scanner using only a focussing lens and the AO deflectors. While this configuration provides for high pixel resolution $(7500 \times 500)$, it is limited in both scan angle $\left(\sim 6^{\circ}\right)$ and in transceiver aperture $(\sim 6 \mathrm{~mm})$. 


\subsection{RESULTS AND DISCUSSION}

\subsection{CLVS System Requirements and Specifications}

The following is a summary of the CLVS system requirements. Table 4-1 provides a summary of CLVS specifications.

Table 4-1. CLVS Specifications

\begin{tabular}{|c|c|c|}
\hline & Phase I & Phase 2 \\
\hline Frame Size & $128 \times 128$ & $256 \times 256$ \\
\hline Frame Rate & one frame per second & one frame per second \\
\hline Working Range & 1.5 -meters & $>1.5$-meters \\
\hline Range Accuracy & $1 \mathrm{~mm}, \mathrm{rms}$ & $1 \mathrm{~mm}, \mathrm{rms}$ \\
\hline Focus & fixed & automatic \\
\hline
\end{tabular}

\section{Requirements}

- Development of a $128 \times 128$ pixel switched fiber-optic 3D vision scanner.

- Development of a fiber-optic CLR transmitter providing linear frequency modulation at the pixel repetition rate. The transmitter was to be based on CRC's existing designs augmented by a fiber optic amplifier to compensate for insertion loses of the switched fiber optic scanner.

- Development of a digital receiver to detect range for each pixel at a $128 \times 128$ pixel frame, one frame per second over a distance of 1.5 meters.

- $\quad$ System implementation using PC or VME based computer hardware (or both). Integrate all CLVS subsystems to achieve performance of the system functional capabilities to acquire and download range image data under direct operator and remote computer control.

- $\quad$ Perform a proof-of-concept baseline system test to demonstrate range accuracy as a function of range and immunity of range measurements to lighting and surface shading.

\section{Success Criteria}

Success criteria for the Base Contract were essentially the specifications listed in Table 4-1. 


\subsection{Test Plan}

The functional and technical test plan and results are documented in "Base Program Interim Phase Test Procedure, Coherent Laser Vision System (CLVS)" dated December 10, 1996. The test plan is summarized in this section. Detailed test results relating to measurement accuracy and sensitivity to surface and lighting characteriztics and signal to noise ratio are to be found in the report; they are summarized in section 4.3 below. Results of the functional demonstration which consisted of viewing real time images produced by the CLVS are discussed in section 4.4 below.

The Bragg cells refract the laser beam proportionally to the frequency of an RF drive signal. Transmission efficiency is linearly proportional to the power of the RF drive signal. The tests were conducted with $3 \mathrm{~W}$ drive power whereas the design predicted that the cells could be run at $6 \mathrm{~W}$. The conservative drive level was used for fear of damaging the Bragg cells with excessive drive power. This issue is discussed in more detail below.

Test procedures and results are discussed below.

\subsection{Test Results}

\subsubsection{Functionality}

Frame size and depth of range were tested by inspection. The frame size is 128 by 128 pixels. The depth of range was observed to by 1.5 meters by operating the system over that depth of range. Frame rate was computed to be 0.9766 frames per second from the pixel period which was measured on an oscilloscope to be 62.5 microseconds. This pixel duration was established by using a $10 \mathrm{MHz}$ clock and a laser drive waveform consisting of 625 data samples. To achieve exactly 1 frame per second with the $10 \mathrm{MHz}$ clock, one needs a pixel duration of 61.03516 microseconds. The system can easily be modified to utilize 610 data samples per pixel which would result in 0.99942 frames per second (1.00058 seconds per frame).

\subsubsection{Range Linearity}

Range linearity was tested by making a series of 31 range measurements at $50 \mathrm{~mm}$ intervals over the $1.5 \mathrm{~m}$ depth of range and examining the error between the measurements and the linear regression straight line fit. A strongly reflecting scotchlight target was used to minimize effects of low signal power. At each target position, 16,384 range measurements were made and the mean and standard deviation of the measurements was calculated. Half of these measurements were made with the laser signal sweeping upward; half were made with the laser sweeping downward. Consequently, 62 mean and standard deviation pairs were computed.

This test identified several problems associated with the hardware. Considering measurements on targets in the near half of the depth of range and measurements on targets in the far half fo the depth of range made when the laser was sweeping downward, range standard 
deviations were all less than $0.3 \mathrm{~mm}$ compared with the $1 \mathrm{~mm}$ requirement. Measurements on targets in the far half of the depth of range made with the laser sweeping upward show larger standard deviations., up to something between 1.0 and $1.2 \mathrm{~mm}$. It was determined that, in certain situations, the special purpose signal processing board reports erroneous results. Since the board could not be returned and repaired within the Base Contract schedule, we chose to accept the results and analyze the errors. Our conclusion is that with repair of the circuit board and some minor modifications to the system software, the large standard deviations will not occur.

Errors between the mean measurements and the linear regression line were less than $1 \mathrm{~mm}$ with the exception of two of the 31 target positions. These two were on the order of $1.5 \mathrm{~mm}$ and they were both in the far half of the depth of range where the results are somewhat suspect as described above.

\subsubsection{Range Accuracy}

Range accuracy and effect of lighting conditions were tested by measuring range to a strongly reflective target at each of five calibrated locations in the depth of range, under different lighting conditions, and with the system having been either off or on for a period of hours before the test. All range errors were less than $1 \mathrm{~mm}$ with the exception of one anomalous value of $3.92 \mathrm{~mm}$. This anomalous error was determined to be the result of an error in the system software.

\subsubsection{Angular pointing Stability}

Pointing stability was tested by pointing the beam at a bream profiler, which measures the location of the peak of the beam. The test was run for a period of 16 hours with a beam measurement being made every five minutes. The results show that the drift in beam pointing over thke 16 hour test amounted to no more than $15 \%$ of the pixel spacing in either direction.

\subsubsection{Angular Pointing Linearity}

Angular pointing linearity was determined by measuring the angular spacing between adjacent pixels for a representative set of points. The spread in the pixel spacing measurements was determined to be $+/-0.002$ degrees out of an angular field of view of approximately 7 degrees, which gives a nominal spacing of 0.05512 degrees between pixels.

\subsubsection{Signal Strength}

Signal strength was used as a measure of optical transmission losses in the Bragg cells. Signal strength was measured directly by measuring power in the beam and indirectly by computing the strength of the measured range signal. Both methods gave similar results which agreed with measurments made by the subcontractor who developed the scanner. Each Bragg cell refracts the beam to 128 angles linearly in one direction. The crossed pair produces the 128 by 128 pointing 
angles. The major effect on signal strength was determined to be a variation of roughly $20 \mathrm{~dB}$ along each direction. This is the main problem that must be overcome in the next design.

\subsubsection{System Losses}

System losses were higher than expected. First, the Bragg cells themselves accounted for between $8 \mathrm{~dB}$ and $9 \mathrm{~dB}$ each, in each direction, for a total of $33.8 \mathrm{~dB}$ attenuation when driven at 3 W. Distortion of the beam shape produced by the Bragg cells accounted for a total of $10 \mathrm{~dB}$. The scan lens itself introduced about $12.8 \mathrm{~dB}$. The total loss turned out to be $56.6 \mathrm{~dB}$, at the point of maximum signal strength. In addition, signal strength fell off by 15 to $20 \mathrm{~dB}$ at the edges of the frame. Consequently, system losses amounted for beteen 56.6 and $76 \mathrm{~dB}$ depending on pixel location. These sources of loss are discussed in turn.

The $12.8 \mathrm{~dB}$ due to the scan lens was expected. An optical lens was chosen instead of a lens coated for IR use to save money, which seemed reasonable at this stage of the development. Future system can utilize a lens optimized for IR usage or the lens could be dispensed with if a smaller angular field of view is acceptable.

Some of the losses are accounted for by the use of $3 \mathrm{~W}$ for Bragg cell drive power. Operating the cells at the design value of $6 \mathrm{~W}$ would improve signal power by $12 \mathrm{~dB}$. It should be noted that the drive power was raised to $6 \mathrm{~W}$ at the end of the laboratory demonstration, with obvious improvement in the quality of the displayed image. Later, however, when we started to take quantitative data at the higher drive power, one of the cells was damaged, conveniently justifying our decision to operate the cells at a conservative drive power.

Losses due to beam distortion, variation in signal strength with pointing angle, and some of the basic loss in the cells can be removed by better design of the Bragg cells and drive transducers. It is estimated that signal strength can be improved by about $37 \mathrm{~dB}$ and the rolloff at the edges of the frame removed.

\subsection{Demonstration}

A program briefing with laboratory demonstration was held at Coleman Research Corp's Springfield Virginia facility on 13 November 1996. The purpose of the laboratory demonstration was to show the real time image display capability under various conditions of range, lighting, and target characteristice. As mentioned before, the Bragg cells were driven at a conservative $3 \mathrm{~W}$. The system displayed reasonably good images at 128 by 128 pixel frames, one frame per second, over a depth of range of 1.5 meters, under lighting conditions ranging from no lights through normal lab lighting to having a spotlight shining directly into the scanner. Strongly reflective targets were used because of the high system losses discussed above.

The final demonstration consisted in showing the effect of Bragg cell drive power. With an image being displayed, the drive power to each cell was reduced from $3 \mathrm{~W}$ to $1.5 \mathrm{~W}$. The result was 
a significantly degraded image. Then the drive power was increased to $6 \mathrm{~W}$. The result was a significantly improved image. Unfortunately, when we tried to obtain quantitative data at $6 \mathrm{~W}$ drive power the next day, one Bragg cell was damaged by the higher power and we could not continue. 


\subsection{CONCLUSIONS AND RECOMMENDATIONS}

\subsection{Conclusions Drawn From the Research Effort}

Table 5-1 summarizes Coleman Research Corporation's achievement of success criteria listed in the Management Plan (Base Contract). In addition, all tasks in the Base Contract, i.e. tasks 1.1 through 1.7 inclusive, have been completed. As was shown in the laboratory, the CLVS meets all functional and technical performance requirements. There were no performance requirements on signal-to-noise (SNR) or image quality, but both were worse than expected, so although the system met all performance requirements, especially range accuracy and repeatability, measurements were made on a very strongly reflective, i.e. scotchlight, target, and real time image quality was not as good as desired. It should be emphasized that the laboratory test was run with the Bragg cells being driven at a very conservative 3 watts. At the end of the test, drive power was increased to 6 watts with noticeable improvement in image quality. We tried to obtain range accuracy test data at the higher drive level after the test but one of the Bragg cells burned out before we could complete the test. In summary, the laboratory system represents a significant step forward in vision systems and no moving parts scanners; all success criteria and performance requirements were satisfied; although SNR and image quality were much poorer than expected, the reasons are understood, and we have every expectation that the next generation of Bragg cell no moving parts scanner will perform as desired. 
Table 5-1. Achievement of Success Criteria

\begin{tabular}{||l|l|l||}
\hline Number & \multicolumn{1}{|c|}{ Criterion } & \multicolumn{1}{|c|}{ Achievement } \\
\hline \hline 1 & $\begin{array}{l}\text { Developed CLVS produces 128 x 128 pixel } \\
\text { frame, one frame per second real-time 3D } \\
\text { images for 5m working range (changed via } \\
\text { contract modification to 1.5 meter depth of } \\
\text { range) }\end{array}$ & $\begin{array}{l}\text { a. } 128 \times 128 \text { pixel frame. } \\
\text { b. One frame per 1.023 seconds } \\
\text { demonstrated; adjustable by count } \\
\text { down factor to one frame per 1.0006 } \\
\text { seconds. } \\
\text { c. Real-time 3D gray scale images } \\
\text { demonstrated. } \\
\text { d. Depth of range of 1.5 meter } \\
\text { demonstrated. }\end{array}$ \\
\hline 2 & $\begin{array}{l}\text { Individual pixel range error approximately } \\
\text { mm rms. }\end{array}$ & $\begin{array}{l}\text { Pixel range error shown by test } \\
\text { procedure to be less than 1 mm for up- } \\
\text { sweeps and down-sweeps for ranges in } \\
\text { the close half of depth of range, and } \\
\text { for down-sweeps in the far half of the } \\
\text { depth of range. Standard deviation } \\
\text { ranges from less than 1 mm to 3 mm } \\
\text { for up-sweeps in the far half of the } \\
\text { depth of range. This has been } \\
\text { attributed to known problems in the } \\
\text { supplier's data processing board and } \\
\text { interface, problems that are easily } \\
\text { fixed. }\end{array}$ \\
\hline \hline
\end{tabular}




\section{APPLICATIONS}

Table 5-2 shows a matrix comparing the CLR vision system with the CLR mapper and with other technology: Amplitude modulated (AM) vision; stereoscopic vision, and structured light. The table clearly points out the unique niche of the FM CLR Vision system for physically small, medium accuracy, high speed mapping that is insensitive to ambient lighting conditions.

Table 5-2. Comparison of Vision Systems

\begin{tabular}{|l|l|l|l|l|l||}
\hline \hline & $\begin{array}{c}\text { FM CLR } \\
\text { Vision }\end{array}$ & $\begin{array}{c}\text { FM-CLR } \\
\text { Mapper }\end{array}$ & AM Vision & \multicolumn{1}{|c|}{$\begin{array}{c}\text { Structured } \\
\text { light }\end{array}$} & \multicolumn{1}{|c|}{$\begin{array}{c}\text { Stereo- } \\
\text { scopic }\end{array}$} \\
\hline \hline $\begin{array}{l}\text { Effect of } \\
\text { ambient light }\end{array}$ & No effect & No effect & Serious & Serious & Moderate \\
\hline Safety & Class I & Class I & Class III & Class III & N/A \\
\hline $\begin{array}{l}\text { Immunity of } \\
\text { range to shading } \\
\text { \& color }\end{array}$ & Yes & Yes & No & No & No \\
\hline Speed & Fast & Slow & Fast & Moderate & Fast \\
\hline Sensor geometry & Single unit & Single unit & Single unit & $\begin{array}{l}\text { Multiple units } \\
\text { with large } \\
\text { baseline }\end{array}$ & $\begin{array}{l}\text { Multiple } \\
\text { sensors }\end{array}$ \\
\hline Range accuracy & Medium & V. High & Low & Medium & Low \\
\hline Angle accuracy & High & V. High & Low & Low & Low \\
\hline Depth of range & Medium & High & High & Medium & Medium \\
\hline Calibration & Auto & Auto & External & Difficult & Difficult \\
\hline Field of view & Small & Large & Large & Large & Medium \\
\hline \hline
\end{tabular}


The main advantage of the CLVS is its ability to produce moderately accurate range maps over a limited volume very fast with automatic calibration and under any and all lighting and surface shading conditions. Major applications are:

- Fast coarse facility mapping, possibly as a prelude to slow, highly accurate, high density mapping by the CLR mapper.

- $\quad$ Fast high density 3D mapping of a restricted volume as encountered in glove box operations, part identification and determination of location and orientation.

- Monitoring a waste stream, utilizing a single dimension scan of the waste stream passing the sensor unit.

The major conclusions to be drawn from Phase I effort are that:

1. The Base Phase success criteria have been met.

2. The basic concept of a solid state no moving parts scanner using two crossed Bragg cell beam refractors providing $1 \mathrm{~mm}$ resolution $3 \mathrm{D}$ frames, at 128 by 128 pixels and at a frame rate of 1 frame per second is feasible.

3. Optical losses in the Bragg cells were higher than expected and this resulted in noisier, i.e., poorer quality, images than expected. The sources of these optical losses have been identified and it is believed that losses can be adequately minimized via design and manufacturing quality control in a second set of Bragg cells.

\subsection{Recommendations for Future Work}

It is recommended that the Continuation Phase option be exercised and that a second set of Bragg cells be fabricated with improved design and fabrication quality control based on what has been learned during the Base Phase. 


\section{Appendix A}

List of Acronyms and Abbreviations 


\begin{tabular}{|c|c|}
\hline Abbreviation(s) & Acronyms \\
\hline $\mathrm{A} / \mathrm{D}$ & Analog/Digital \\
\hline $\mathrm{AO}$ & Acousto-optic \\
\hline $\mathrm{AZ}$ & Azimuth \\
\hline CLR & Coherent Laser Radar \\
\hline CLVS & Coherent Laser Vision System \\
\hline CRC & Coleman Research Corporation \\
\hline CPU & Central Processing Unit \\
\hline $\mathrm{dB}$ & Decibel \\
\hline DFB & Distributed feedback \\
\hline DOE & Department of Energy \\
\hline DSP & Digital signal processing \\
\hline EL & Elevation \\
\hline FFT & Fast Fourier Transform algorithm \\
\hline $\mathrm{FM}$ & Frequency modulated \\
\hline $\mathrm{GHz}$ & Gigahertz \\
\hline GUI & Graphical user interface \\
\hline I\&Q & In-phase \& quadrature \\
\hline IR & Infrared \\
\hline LO & Local oscillator \\
\hline $\mathrm{MHz}$ & Megahertz \\
\hline $\mathrm{OS} / 2$ & IBM Operating System 2 \\
\hline $\mathrm{PC}$ & Personal computer \\
\hline $\begin{array}{l}\mathrm{PC} / \mathrm{VME} \\
\text { pinFET }\end{array}$ & $\begin{array}{l}\text { PCI (Peripheral Computer Interface) bus to VMEbus Interface } \\
\text { positive intrinsic negative Field-Effect-Transistor }\end{array}$ \\
\hline RF & Radio frequency \\
\hline RS-170 & Video signal standard \\
\hline TBD & To be determined \\
\hline TBR & To be reviewed \\
\hline TBS & To be supplied \\
\hline $\mathrm{TeO}_{2}$ & Tellurium dioxide \\
\hline UTP & Uniphase Telecommunications Products \\
\hline VSWR & Voltage standing wave ratio \\
\hline VME & Computer bus standard \\
\hline $2 \mathrm{D}$ & Two-dimensional \\
\hline $3 \mathrm{D}$ & Three-dimensional \\
\hline
\end{tabular}


Appendix B

Statement of Work 


\section{STATEMENT OF WORK Coherent Laser Vision System}

\section{A. OBJECTIVE}

The objective of the proposed research effort is to develop a prototype fiber optic based coherent last vision system (CLVS) suitable for DOE's EM Robotics program. The CLVS will provide three dimensional (3D) vision for monitoring situations in which it is necessary to update geometric data on the order of once per second. Further, based upon digitized data whole 3D scene views will become available on a real-time basis.

The CLVS shall be developed upon the contractor's proprietary fiver optic coupled frequency modulated continuous wave coherent laser radar technology. The radar uses the relatively large tuning range of injection laser diodes to achieve greater precision than available with other techniques. The CLVS shall implement innovations in fiber optic multiplexed scanning and digital receiver design which yield improved range accuracy with reduced receiver processor loading. The project will integrate commercially available state-of-the-art fiber optic components, such as an optical amplifier.

\section{B. SCOPE OF WORK}

The program shall be implemented in two components; a Base Contract and a continuance Option. In the Base Contract, the contractor shall develop a prototype coherent laser radar vision system, CLVS, with a baseline frame density of $128 \times 128$ pixels, one frame per second with 1.5 meter depth of range; achieve projected accuracy over the full operating range; and achieve stable scanner beam pointing for each pixel. The CLVS shall be capable of demonstrating total immunity of $3 \mathrm{D}$ position estimates to ambient lighting, color and surface shading.

In the continuance Option, the contractor shall develop a compact scanner and implement advanced receiver algorithms to achieve a fieldable prototype coherent laser radar 3D vision system. The objectives are to increase the prototype CLVS frame density to one $256 \times 256$ frame per second real-time performance; increase the operating range beyond 5 meters; achieve this increased performance through the use of a higher density fiber optic-based scanner and a "smart receiver" digital pixel processing approach; and finally, perform functional system tests and test the end product as a practical, fieldable system for use in the EM robotics program.

The software work shall be compatible with the Generic Intelligent System Control (GISC). The GISC was developed by the Sandia National Laboratory to integrate simple subsystems to achieve complex capabilities, automate operations using model-based control, and stress modularity to achieve compatibility for robotics systems. 


\section{TASKS TO BE PERFORMED}

\section{Base Contract}

\section{Task 1.1: Switched Fiber Optic 3D Vision Scanner Development}

The contractor shall design and develop a $128 \times 128$ pixel switched fiber optic 3D Vision Scanner which accomplishes 2D scanning with no moving parts.

\section{Task 1.2: Fiber Coupled Laser Transmitter and Optical Amplifier Development}

The contractor shall develop a fiber optic CLR transmitter which provides linear frequency modulation at the pixel repetition rate. The transmitter shall be based upon the contractor's existing designs augmented by a fiver optic amplifier as necessary to overcome insertion losses of the scanner.

\section{Task 1.3: Digital Receiver Development}

The contractor shall develop a digital receiver which detects range for each pixel at a $128 \mathrm{x}$ 128 pixel frame, one frame per second rate over a working range of 1.5 meters.

\section{Task 1.4: Host Computer and Monitor, Graphical User Interface, External Data Interface Development}

The contractor shall install, debug, and implement a system control and graphical user interface software. They shall implement GISC compatible external control software on a hardware interface which is GISC compatible. They shall develop hardware interfaces to download digital range image data in both standard video interface (RS-170) and digital video formats.

\section{Task 1.5: Baseline CLVS System Integration}

The contractor shall integrate all CLVS subsystems to achieve performance of the system functional capabilities to acquire and download range image data under direct operator and remote computer control.

\section{Task 1.6 Baseline System Test -- Proof-of-Concept}

The contractor shall perform, a real-time CLVS functional performance test, including range accuracy as a function of range and immunity of range measurements to lighting and surface shading. The contractor shall establish maximum acceptable tolerances on the standard deviation of measurement errors throughout the CLVS range. These tolerances shall be specified on angular (azimuth and elevation) and radial measure. In addition, a specification 
of what constitutes "total immunity to 3D position estimates to ambient lighting, color and surface shading" shall be established.

This task is simply to show DOE "proof-of-concept" before the Government proceeds to exercise the continuance Option of the contract. The contractor shall establish minimum testing procedures and standards (plan) to verify that the prototype meets design specifications. These procedures and standards shall be presented to DOE COR in a Test Report, prior to the systems test.

\section{Task 1.7: Interim Report}

The contractor shall prepare and submit for review and comment an interim phase Topical Report on the project's activities including test results at least 60 days before completion of Base Contract efforts. This shall be a stand-alone document detailing all activities including, but not limited to, details and results of the systems engineering analyses, CLVS system specifications, drawings of the CLVS system, projected system performance parameters, description of test activities and presentation of test results, data illustrating the capabilities of the CLVS, data collection methods and processing techniques applied, demonstration results, conclusions drawn from the research effort, and recommendations for future work. This document shall also note any unusual features discovered that would impact future progress of the technology. DOE will advise the contractor of acceptance of the report or the need for revisions. The DOE will provide its comments within 30 days after submission of the draft report.

Should DOE decide not to exercise the option, then the interim Topical Report will be advised to become the Final Report.

\section{Option}

\section{Task 2.1: Compact Switched 3D Vision Scanner Development}

The contractor shall design and develop a $256 \times 256$ pixel no moving parts 3D Vision Scanner. This scanner shall be a compact, fiber optic tethered implementation which can be operated remotely from the CLVS transmitter/processor unit.

\section{Task 2.2: Smart Receiver Software Algorithm Development}

Based upon results and experience gained from Base Phase efforts, the contractor shall generate "smart receiver" algorithms which allow a receiver to compute range images more efficiently by processing less than the whole signal bandwidth for each pixel through the use of a priori image knowledge. This smart receiver shall be designed to provide real-time update of all range pixels while reducing processing load substantially below blind full range processing of all pixels. 
The contractor shall conduct a test of the CLVS system at the selected DOE site, as outlined in the Test Plan above. The contractor shall coordinate date, time, activities, list of participants, and provide for any necessary clearances or permits, training, support services, personnel security clearances for the test demonstration with the DOE/METC COR, sixty (60) days prior to performance. After the completion of the tests, the contractor shall perform an evaluation of results and if applicable discuss inconsistencies between laboratory and field tests. This evaluation shall be presented in the final report, Task 2.8 .

\section{Task 2.8: Final Report}

The contractor shall prepare and submit for review and comment a draft final report on this project's activities sixty (60) days before the contract performance period ends. This shall be a stand-alone document describing all activities including, but not limited to, details and results of the systems engineering analyses, CLVS system specifications, drawings of the CLVS system, projected system performance parameters, description of test activities and presentation of test results, data illustrating the capabilities of the CLVS system, data collection methods and processing techniques applied, demonstration results, conclusions drawn from the research effort, and recommendations for future work. This document shall also note any unusual features discovered that would impact future progress of the technology. This report shall provide DOE with sufficient information for dissemination of results to interested DOE programs, offices and sites. The DOE will provide its comments within 30 days after submission of the draft final report, advising the contractor of acceptance or the need for revisions. The contractor shall submit a final report within 30 days of notice of acceptability of the draft report.

\section{BRIEFINGS}

The contractor shall prepare two detailed briefings for presentation to the DOE METC/COR. These briefings shall be given by the contractor to explain the plans, progress, and results of the technical effort. One briefing shall be given after the completion of the Base Contract system test, Task 1.6 -- the decision point to exercise option of continuance. The second briefing shall follow completion of field tests, Task 2.7, in the Option component of the contract to explain the final results of the research effort. In addition, the contractor shall prepare for two Kick-off meetings to be held in Morgantown, WV. The purpose of these meetings are for post award briefing for DOE/EWM of the contractor's proposed project management (see MANAGEMENT PLAN clause.) These meeting shall occur prior to start of each contract component, i.e., Base Contract and Option.

The contractor shall also prepare and present briefings for the annual Office of Technology Development meeting generally held in Washington, D.C. 


\section{E. DELIVERABLES}

The contractor shall submit General Management, Schedule/Labor/Costs, Exception, Technical and other periodic reports in accordance with the Contract Reporting Requirements Checklist.

The contractor shall submit the following Topical Reports, referred to in the Statement of Work:

a. $\quad$ Baseline Test Report, described in Task 1.6.

b. Base Program Interim Phase Topical Report, described in Task 1.7.

c. - CLVS Systems Operations/Users Manual(s) and Field Test Site Plans Report, described in Tasks 2.5 and 2.7 .

d. Draft and final report, described in Task 2.8.

If the continuance Option is not exercised for Option work, the topical report submitted in the Base Phase shall be considered the final report.

The contractor shall deliver to DOE a complete operating fieldable prototype Coherent Laser Radar 3D Vision System after the completion of tests. The system shall include but not be limited to: hardware, software, users/operations manual and training documentation if required. 